\title{
Essai sur la carte de G. Gastaldi « Brasil » insérée dans « Delle navigationi et Viaggi » de G. B. Ramusio (1556; $1565 ; 1606)$
}

Ensaio sobre o mapa «Brasil» de G. Gastaldi inserido em Delle navigationi e viaggi de G. B. Ramusio (1556; 1565; 1606)

Ensayo sobre el mapa «Brasil» de G. Gastaldi inserto en el Delle navigationni e Viaggi de G. B. Ramusio (1556; 1565; 1606)

Essay on the map of Brazil by G. Gastaldi included in G. B. Ramusio's Delle

Navigationi et Viaggi $(1556 ; 1565 ; 1606)$

\section{Olga Okuneva}

Traducteur : Paul Sutermeister

\section{(2) OpenEdition}

\section{Journals}

Édition électronique

URL : https://journals.openedition.org/terrabrasilis/743

DOI : 10.4000/terrabrasilis.743

ISSN : 2316-7793

Éditeur

Rede Brasileira de História da Geografia e Geografia Histórica

\section{Référence électronique}

Olga Okuneva, «Essai sur la carte de G. Gastaldi «Brasil » insérée dans «Delle navigationi et Viaggi » de G. B. Ramusio $(1556$; 1565 ; 1606) », Terra Brasilis [En ligne], 2 | 2013, mis en ligne le 21 juin 2013, consulté le 05 décembre 2022. URL : http://journals.openedition.org/terrabrasilis/743 ; DOI : https:// doi.org/10.4000/terrabrasilis.743

Ce document a été généré automatiquement le 5 décembre 2022.

Tous droits réservés 


\section{Essai sur la carte de G. Gastaldi « Brasil » insérée dans « Delle navigationi et Viaggi » de G. B. Ramusio $(1556 ; 1565 ; 1606)$}

Ensaio sobre o mapa «Brasil» de G. Gastaldi inserido em Delle navigationi e viaggi de G. B. Ramusio (1556; 1565; 1606)

Ensayo sobre el mapa «Brasil» de G. Gastaldi inserto en el Delle navigationni e Viaggi de G. B. Ramusio (1556; 1565; 1606)

Essay on the map of Brazil by G. Gastaldi included in G. B. Ramusio's Delle Navigationi et Viaggi $(1556 ; 1565 ; 1606)$

\section{Olga Okuneva}

Traduction : Paul Sutermeister

\section{NOTE DE L'ÉDITEUR}

Ofeceremos neste mesmo número uma tradução ao português deste artigo sob o título Ensaio sobre o mapa «Brasil» de G. Gastaldi pertencente ao Delle navigationi e viaggi de G. B. Ramusio $(1556 ; 1565 ; 1606)$

Ofrecemos en este mismo número una traducción al portugués de este artículo con el título Ensaio sobre o mapa «Brasil» de G. Gastaldi pertencente ao Delle navigationi e viaggi de G. B. Ramusio $(1556 ; 1565 ; 1606)$

Nous offrirons dans cet même numéro une traduction en Portugaise de cet article sous le titre Ensaio sobre o mapa «Brasil» de G. Gastaldi pertencente ao Delle navigationi e viaggi de G. B. Ramusio $(1556 ; 1565 ; 1606)$ 
We offer in this same issue a Portuguese translation of this paper under the title Ensaio sobre o mapa «Brasil» de G. Gastaldi pertencente ao Delle navigationi e viaggi de G. B.

Ramusio $(1556 ; 1565 ; 1606)$

1 La Bibliothèque digitale de la cartographie historique - une excellente initiative de l'Université de São Paulo qui met à la disposition des chercheurs des cartes anciennes numérisées d'une très haute qualité - abrite dans sa collection une carte intéressante du Brésil du XVI ${ }^{\mathrm{e}}$ siècle ${ }^{1}$.

Figure 1: Giacomo Gastaldi - Brasil (1556)

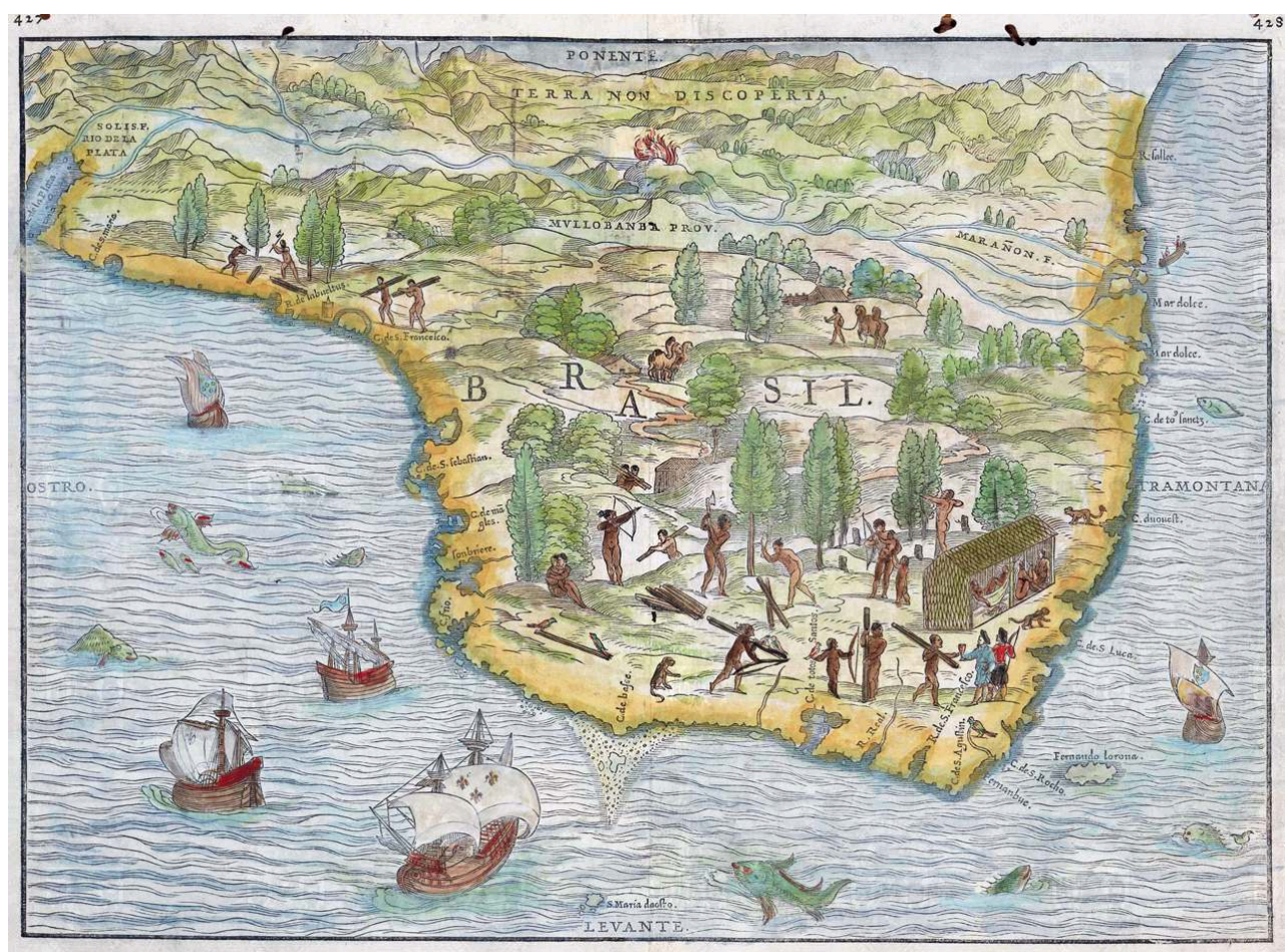

In: Giovanni Battista Ramusio, Delle Navigationi et Viaggi (1556).

Exemplaire coloré de la Bibliothèque Numérique de Cartographie Historique - Université de São Paulo [http://www.cartografiahistorica.usp.br/index.php?

option=com_jumi\&fileid=14\&ltemid=99\&idMapa=579].

2 Cette carte accompagne une curieuse relation sur le Brésil de la première moitié du $\mathrm{XVI}^{\mathrm{e}}$ siècle : le texte apparaît dans le troisième volume des "Navigations et voyages » de Giovanni Battista Ramusio ${ }^{2}$. Ce Vénitien - diplomate, humaniste et grand amateur de la géographie - a rassemblé plusieurs ouvrages géographiques et relations de voyages dans un recueil en trois volumes. Tout en restant au service de l'Etat vénitien pendant une grande période de sa vie (d'abord attaché à la chancellerie, ensuite en tant que secrétaire du Sénat et un des secrétaires du Conseil de Dix), il a collaboré également à l'administration pratique d'une grande collection de manuscrits (le noyau de la future Biblioteca Marciana), ce qui atteste non seulement son intérêt pour la culture livresque mais aussi une habilitation à mener un travail «de routine » dans la gestion de la collection ${ }^{3}$.

3 Le propre intérêt de Ramusio en littérature géographique, en relations manuscrites ou imprimées de voyages contemporains et en livres sur les découvertes géographiques «faites en notre siècle» est inséparable de sa passion pour les lettres et pour les 
auteurs de l'antiquité classique. C'était dans cet esprit de la Renaissance et d'humanisme et dans le contexte de liens étroits avec des humanistes, tels que Pietro Bembo, Andrea Navagero et Girolamo Fracastoro, que l'œuvre principale de Ramusio sa grande collection de textes géographiques - est née.

4 A l'époque où le prestige de la "Géographie » de Ptolémée est déjà ébranlé par les découvertes maritimes, on cherche à combiner les informations de cet auteur avec les observations des voyageurs modernes et de réviser ainsi les modeles classiques. Telle est la démarche de Waldseemüeller en 1511 et en certaine partie en 1513; la même pensée guide Ramusio dans son désir de fournir au public les relations originelles des voyageurs, riches en détails et en observations, pour élaborer à leur base de nouvelles cartes ${ }^{4}$.

de ceux qui ont contribué à ce dessein est le cartographe Giacomo Gastaldi, Vénitien d'origine piémontaise et le cosmographe officiel de la Sérénissime République. Une bonne connaissance entre Ramusio et Gastaldi s'accroit à la fin des années 1540 lorsque celui-ci commence à enseigner la géographie au fils de Ramusio ${ }^{5}$. Il est probable que l'exécution par Gastaldi d'une grande commande officielle - dans les années 1540 il a été chargé de refaire les anciennes cartes régionales du $\mathrm{XV}^{\mathrm{e}}$ siècle sur les murs d'une salle au Palais Ducal - se doive aux informations que le cartographe avait puisé dans la collection de Ramusio.

C'est Giacomo Gastaldi qui est l'auteur d'une carte gravée du Brésil qui sera analysée dans le présent article. Gastaldi a fait douze cartes pour le recueil de Ramusio : trois sont publiées dans le premier volume (Afrique) et neuf dans le troisième (dont sept se réfèrent à l'Amérique). En dehors du travail pour Ramusio, Gastaldi a produit un nombre de cartes individuelles, parfois commandées par les mécènes ${ }^{6}$.

7 Le grand ensemble éditorial que présentent les trois volumes de "Navigations et voyages " garde visible le travail de l'éditeur en matière de choix de textes, de leur traduction en italien afin de contribuer à la cohérence de la collection et en commentaires. Ces principes peuvent être observés sur l'exemple du texte sur le Brésil accompagné par la carte de Gastaldi.

8 La relation qui fait partie du troisième volume dédié au Nouveau Monde est intitulée "Discorso d'un gran capitano di mare francese del lucco di Dieppa sopra le navigationi fatte alle Terra Nuova dell'Indie Occidentali, chiamata la Nuova Francia, da gradi 40 fino a gradi 47 sotto il Polo Arctico, \& sopra la terra del Brasil, Guinea, Isola di San Lorenzo,\& quelle di Sumatra, fino alle quali hano navigato le caravelle \& navi francese"7. Le document est publié en italien mais le texte original a été rédigé en français et Ramusio indique que c'est lui qui a voulu le retransmettre «en notre langue $»^{8}$. La traduction a pu être faite par Ramusio lui-même, car sa maîtrise du français (tirée probablement d'une mission diplomatique en France) lui a valu des éloges des contemporains et l'exercice de la charge de l'interprète $d u$ français auprès $d u$ doge ${ }^{9}$. Il est bien probable que Ramusio ait commandé lui-même les nouveaux matériaux (dont le « Discours ») de Paris à la fin des années 1530 et au début des années $1540^{10}$.

9 En incluant cette relation dans son ouvrage, Ramusio ignorait le nom du "grand capitaine » et de l'auteur du "Discours» (en effet, il pensait qu'il s'agit d'une seule personne; nous reviendrons à cette question ci-dessous). Le fait de méconnaître son nom lui paraissait cependant un grand dommage: "Ce discours est vraiment très beaux et digne d'être lu par chacun, mais nous sommes désolés de ne pas savoir le nom 
de l'auteur car, son nom n'y étant mis, ce sera injure à la mémoire d'un si vaillant et noble gentilhomme $»^{11}$.

La relation du voyage et le voyage même restaient anonymes jusqu'aux années 1830 où un manuscrit d'un journal de bord d'une expédition française à Sumatra en 1529 sous le titre provisoire « Voyage des Dieppois » a été trouvé dans les papiers particuliers d'un négociant à Rouen. Tenant compte du fait que le « Discours d'un grand capitaine » parle de Sumatra dans les termes semblables et que le journal de bord indiquait clairement le nom du capitaine français ayant entrepris ce voyage - Jean Parmentier, aussi bien que le nom du narrateur - Pierre Crignon, l'attribution a été faite. L'historien français Louis Estancelin, à l'origine de cette attribution, affirmait en 1832 :

11 La découverte que le hasard nous a procurée du journal [du voyage à Sumatra] ne laisse plus aucune doute...Il convenait de publier en entier le journal et la relation donnée par Ramusio ; ces deux pièces, se prêtant un mutuel appui, concourent à ne laisser aucun doute sur la réalité et l'identité du voyage qu'elles décrivent ${ }^{12}$.

Il s'agit ainsi d'une expédition de 1529 conduite par Jean et Raoul Parmentier sur les deux navires, le «Sacre » et la «Pensée ». La visite du Brésil, s'inscrivait-elle dans le cadre de ce voyage? Ramusio, le publicateur du «Discours d'un grand capitaine", semblait le croire :

13 [Le grand capitaine français] décrit le voyage qui se fait à la terre neuve des Indes Occidentales, que nous appelons aujourd'hui la Nouvelle France, et aussi à la terre du Brésil des dites Indes, en Guinée et à la côte des Malaguettes sur la côte de l'Afrique, que les Français fréquentent tous les jours avec leurs navires. Le susdit capitaine ensuite, avec deux navires armés à Dieppe en Normandie, sut aller jusqu'à l'île de Taprobane dans l'est, aujourd'hui dite Sumatra, où il négocia avec les gens; et, chargés d'épices, il rentra chez lui ${ }^{13}$.

14 Cependant une question se pose : Ramusio affirme que le «Discours » a été fait en $1539^{14}$; or, Jean Parmentier est mort avant 1531 ou 1532, comme en témoigne le recueil de ses poèmes publiés post mortem par son compagnon Pierre Crignon qui déplore la mort de Jean et de Raoul Parmentier ${ }^{15}$. De plus, le texte du « Discours... » contient un important marqueur chronologique : «Une partie de cette terre du Brésil fut d'abord découverte par les Portugais, et il y a environ trente-cinq ans $»^{16}$. Jean Parmentier ne pourrait pas ainsi être l'auteur du «Discours", comme pensait Ramusio ; par contre, son ami et compagnon de voyage Pierre Crignon parait bien l'auteur en question. Il a déjà relaté le voyage à Sumatra; l'affection qu'il portait à la mémoire des frères Parmentier et qui l'a poussé à éditer les poèmes de Jean Parmentier conjugue bien avec le souci de commémorer les voyages de celui-ci dans un mémoire synthétique.

La paternité de Crignon est depuis lors reconnue dans l'historiographie française ${ }^{17}$. Il existe cependant d'autres versions, telles que l'attribution du texte au cosmographe français d'origine portugaise Jean Alphonse. Encore une hypothèse consiste à voir en personne de Jean Alphonse non pas l'auteur du «Discours..." mais l'auteur de la "Cosmographie » dont les passages ont inspiré Ramusio qui les aurait assigné à un certain capitaine ${ }^{18}$.

7 Après avoir présenté l'œuvre de Ramusio et les discussions sur la personnalité de l'auteur du "Discours", passons à l'examen de la carte de Gastaldi qui vient avec le texte. Il existe trois éditions du troisième volume des « Navigations et voyages » (et 
donc de la carte en question) - celle de 1556, de 1565 et de 1606 (notons que ce troisième volume sur l'Amérique a connu moins de rééditions que les autres volumes de l'ensemble ${ }^{19}$ ). Une édition de 1565 reprend la mise en page de la première édition de 1556 et ainsi la carte se trouve dans ces deux cas aux pages 427-428, à la différence de l'édition de 1606 où elle est aux pages 356-357. A partir de cette observation il serait aisé de supposer que les variantes de 1556 et de 1565 sont plutôt identiques et peuvent différer de la variante de 1606. Pourtant la situation est inverse : la comparaison de trois variantes ${ }^{20}$ montre que les exemplaires de 1565 et de 1606 sont pareils, tandis que celui de 1556 est légèrement différent. Les différences sont de trois types :

- typographiques (police plutôt arrondie en 1556, plus aiguë en 1565 et 1606 ; le format des chiffres qui composent le numéro de page 427 ; l'existence ou l'absence d'espace entre les mots dans les inscriptions « Rio de la Plata » et «Mar dolce »; l'existence ou l'absence de point après les toponymes) ;

- iconographiques (la position et la configuration des tracées qui symbolisent le relief du pays diffèrent systématiquement; le personnage à la hache dans la partie centrale de la carte tient son outil tourné à gauche en 1556 et à droite en 1565/1606; le personnage portant les troncs d'arbres en tient deux en 1556 et seulement un en 1565/1606; le tireur à l'arc est du sexe incertain en 1556 et devient une femme aux seins bien marquées en 1565/1606; la queue d'un singe en bas de la carte est tournée à gauche en 1556 et à droite en 1565/1606 etc.)

- orthographiques et de conservation : l'état de sauvegarde de trois exemplaires de la carte $(1556,1565$ et 1606) et les conditions de leur digitalisation sont différents. Ainsi, dans l'exemplaire de la bibliothèque de Heidelberg la feuille avec la carte a été pliée de telle manière que même lorsqu'elle est dépliée, certains petits éléments sont «mangés » par le pli. Cela se réfère surtout aux inscriptions qui perdent une lettre : "Mulloban(b)a prov.", "Terra no(n) descoperta", "Ponen(t)e", "Leva(n)te" etc. Toutefois une véritable différence d'orthographe oppose encore une fois la variante de 1556 et ceux de 1565/1606: dans le premier cas on lit "Terra non discoperta" et dans le second "Terra non descoperta".

Les trois variantes de cartes tirées des éditions de 1556, de 1565 et de 1606 sont en noir et blanc. Un exemplaire de la Bibliothèque digitale de la cartographie historique de l'Université de São Paulo est coloré, mais il est difficile de dire de quelle époque date la coloration (la partie gravée est bien de 1556, comme le montre la comparaison avec l'exemplaire de la bibliothèque de Heidelberg). On peut seulement constater que la coloration de l'exemplaire de l'USP ne suit pas la tradition - pourtant bien répandu au $\mathrm{XVI}^{\mathrm{e}}$ siècle - de montrer d'une manière différente les arbres ordinaires et les troncs des arbres pau brasil (il en sera question ci-dessous).

Comparons maintenant les éléments essentiels de la carte et du texte. 
Figure 2: Giacomo Gastaldi - Brasil (1556)

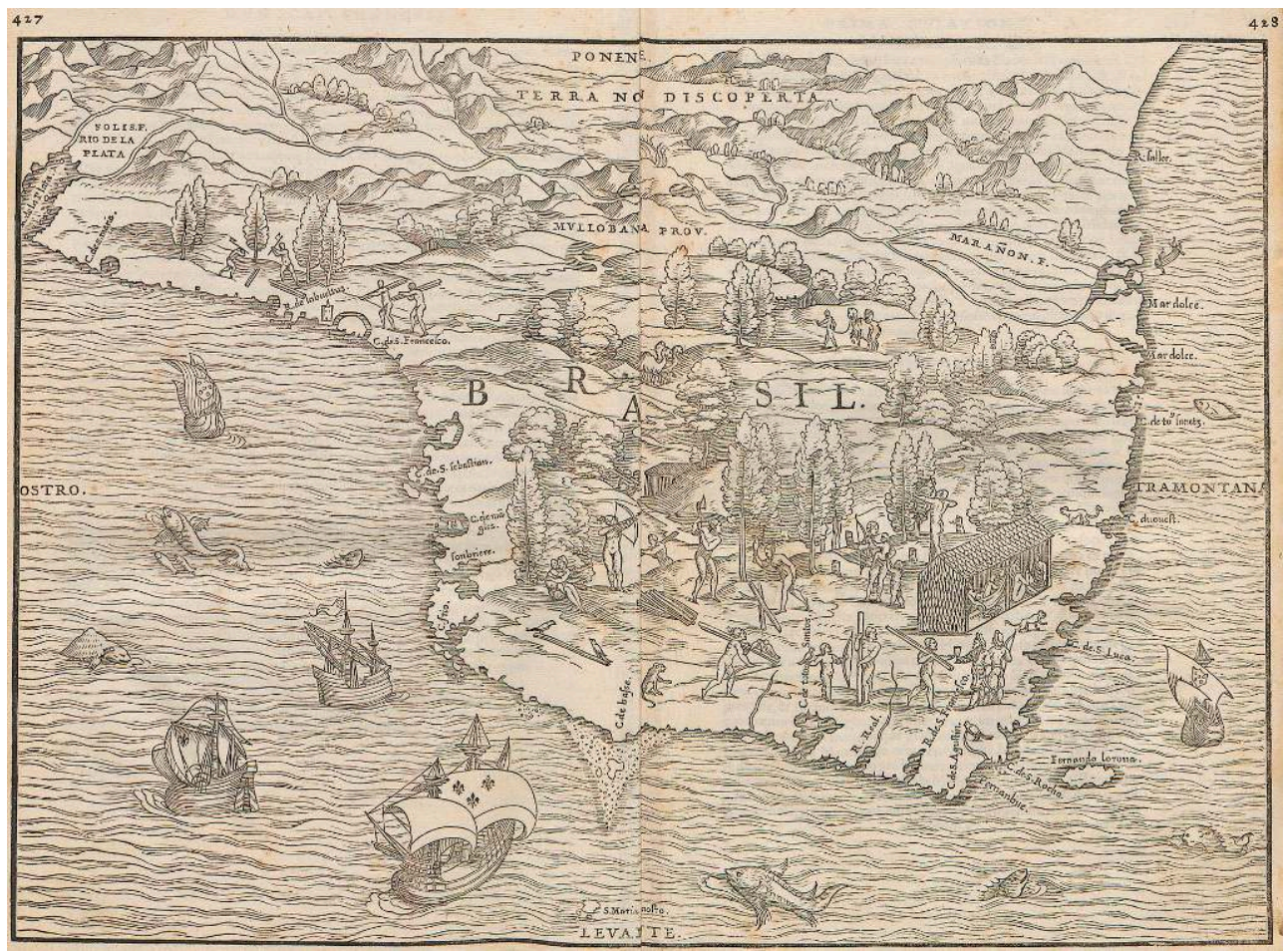

IN: GIOVANNI BATTISTA RAMUSIO, DELLE NAVIgATIONI ET VIAggI (1556)

Exemplaire de la Bibliothèque de l'Université de Heidelberg [http://digi.ub.uni-heidelberg.de/diglit/ ramusio1556bd3/0936] 
Figura 3: Giacomo Gastaldi - Brasil (1565)

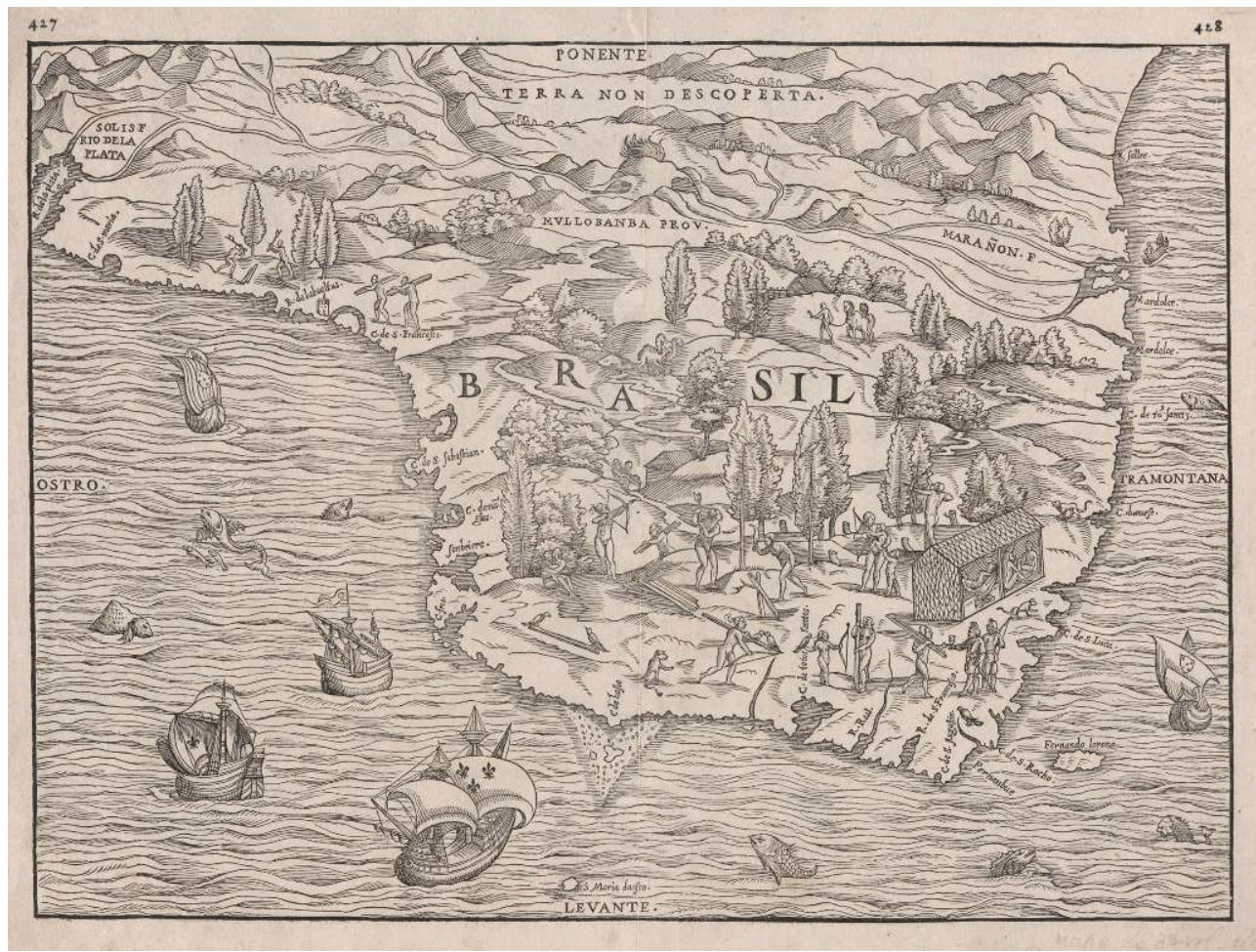

IN: GIOVANNI BATTISTA RAMUSIO, DELLE NAVIgATIONI ET VIAggI (1565)

Exemplaire de la Bibliothèque Nationale du Brésil [http://objdigital.bn.br/acervo_digital/ div_cartografia/cart395872.jpg] 


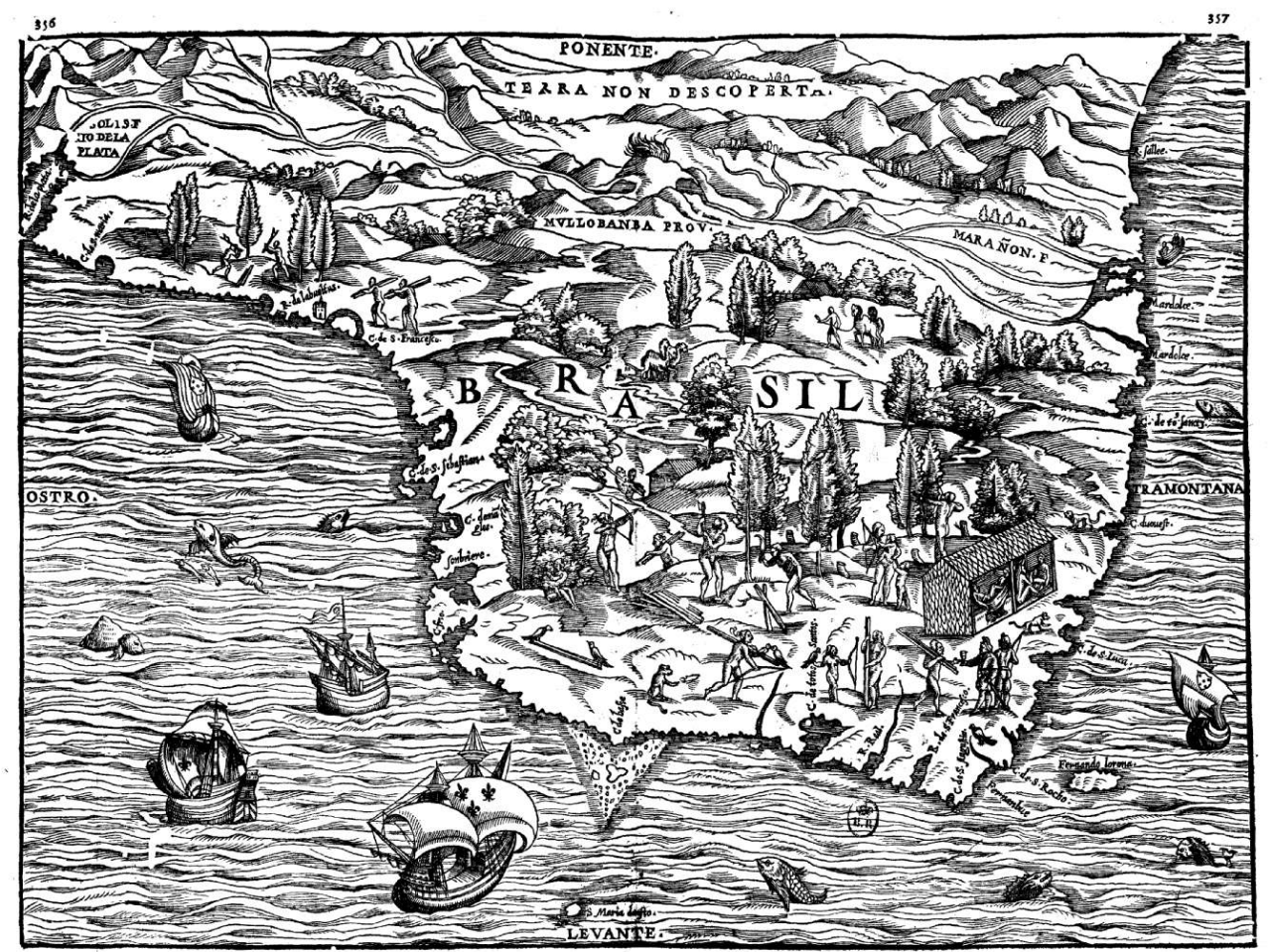

IN: GIOVANNI BATTISTA RAMUSIO, DELLE NAVIgATIONI ET VIAggI (1606)

Exemplaire de la BNF-Gallica [http://gallica.bnf.fr/ark:/12148/bpt6k131851r/f791.image]

\section{La présentation générale du Brésil et la nomenclature géographique}

20 En examinant la présentation générale du Brésil proposée par le texte et la carte, on constate aussi bien la similitude que le décalage. Ainsi, le texte parle de la terre du Brésil dont les extrémités sont le cap de Onze mille Vierges (actuel Cabo Virgines) près du Détroit de Magellan et le fleuve Maragnon (Amazone); la longueur de cette côte est une somme de distances entre les points de repère les plus importants (depuis le cap de Onze mille Vierges - Rio de la Plata, cap de Sainte Marie, cap du Saint-Augustin, cap du Saint Roch, golfe de Saint-Luc, cap du Couchant, fleuve du Maragnon). Au-delà de ce fleuve, statue Pierre Crignon, on rencontre les îles et les terres découvertes par les Espagnols dans les Indes occidentales ${ }^{21}$. En même temps cette présentation du littoral ne touche point les régions intérieures du Brésil.

21 En comparaison avec le "Discours" la carte offre une autre vision du Brésil. Tout d'abord elle est orientée selon l'axe occident-orient ce qui n'est pas rare à l'époque ${ }^{22}$; la ligne du littoral atlantique va ainsi de gauche à droite et c'est ici qu'on constate une différence par rapport au texte : en gardant le fleuve de Maragnon en tant qu'une des limites naturelles du pays, la carte présente comme une autre extrémité non plus le cap de Onze mille Vierges mais le Rio de la Plata (la légende de la carte associe à ce toponyme de nom de Juan Diaz Solis en hommage à sa découverte de 1516).

D'autre côté Gastaldi ne se limite pas de montrer la frange atlantique du pays mais représente également la masse du continent. Bien que ces zones restent méconnues et 
ne portent aucune légende sauf « Mullobanba prov. » et "Terra non descoperta », on y remarque une silhouette d'un volcan en pleine éruption. De deux côtés du volcan le cartographe a placé des lacs où prennent naissance les deux grands fleuves mentionnés ci-dessus - Rio de la Plata et Maragnon. Gastaldi participe ainsi à une longue discussion cartographique sur l'existence d'un certain lac au centre du continent où prennent naissance les deux fleuves qui se trouvent ainsi liées et qui font du Brésil une gigantesque île. Cette thèse de l'insularité du Brésil, présente chez certains cartographes du XVI ${ }^{e}$ siècle, a acquis un caractère politique au XVII ${ }^{e}$ siècle pour contribuer à la démarcation des possessions portugaises et espagnoles en Amérique du $\mathrm{Sud}^{23}$. On voit que Gastaldi ne partageait pas l'opinion de l'insularité du Brésil, bien qu'il ait représenté les lieux de naissance des deux grands fleuves assez proches l'un d'autre.

Quant à la nomenclature géographique, les similitudes entre la carte et le texte sont assez visibles. La carte contient tous les toponymes mentionnés dans le texte (à l'exception du cap de Onze mille Vierges et le détroit de Magellan) et en représente encore une dizaine d'autres. De tous les toponymes ce sont les caps qui sont en majorité ; ensuite viennent les rivières. Un des deux toponymes figurant sur la carte sans une abréviation « $\mathrm{C}$.» (cap) ou «R.» (rivière) attire une attention particulière. C'est le Fernambuc (Pernambuco) qui a été spécialement évoqué dans le « Discours d'un grand capitaine » en tant qu'un seul point fort des Portugais sur le littoral :

Le cette côte et vers le couchant, les Portugais n'ont élevé aucun château ou forteresse ; seulement, on trouve dans un lieu dit Fernambuc, situé après le cap SaintAugustin, une petite forteresse de bois avec peu de gens exilés du Portugal ${ }^{24}$.

25 Il est intéressant de constater que la mention de "Fernambuc " ( Fernanbuch » dans le texte de Crignon) sur la carte n'est pas accompagnée par quelconque dessin ou pictogramme évoquant une ville (une tour ou un château, un clocher ou une cathédrale). Cependant un pictogramme pareil semble être présente sur la carte, audessus du Cap Saint-François, mais aucun toponyme « urbain » ne le suit. En tout cas l'exemple du « Fernambuc » montre que la carte publiée dans les années 1550 ne reflète pas les réalités récentes du développement urbain du Brésil ${ }^{25}$; c'est ici qu'elle correspond au texte datant de la période précédente, et le texte, à son tour, remontre au voyage à l'époque encore plus éloignée.

\section{La présence des étrangers et la rivalité franco- portugaise au Brésil}

26 Il est bien connu qu'à la Renaissance la carte géographique joue plusieurs rôles, et la fonction d'un instrument politique et diplomatique n'en est pas le moindre ${ }^{26}$. Ainsi, les cartes affichent les pavillons et les blasons des souverains et des grands seigneurs, portent des dédicaces aux grands de ce monde etc. La carte du Brésil de Gastaldi n'est pas libre, non plus, des références politiques. L'analyse de leurs particularités serait incomplet sans tenir compte du texte que la carte illustre et accompagne.

$27 \mathrm{Au}$ premier regard la représentation du Brésil par Gastaldi est "politiquement neutre » : aucun blason n'est placé dans les contours du pays, aucune légende faisant référence à la nationalité des découvreurs ou des souverains n'est présente. Et pourtant le Brésil de Gastaldi fait bien l'objet de convoitise : c'est en dehors des contours du pays 
que les références politiques et diplomatiques apparaissent. Il s'agit des armoiries portées par des nombreux navires qui vont et viennent le long des côtes brésiliennes.

Les cinq navires visibles sur la carte sont tous différents. Des deux folios occupés par la carte c'est la partie gauche qui concentre la majorité des navires (quatre); la partie droite n'en compte qu'un seul. Parmi tous les navires on voit deux aux fleurs de lys français et trois autres aux armoiries portugaises. Ceux-ci sont peints de trois côtés du Brésil, dans un périmètre de l'espace aquatique du pays. Les vaisseaux français sont représentés en dehors par rapport aux navires portugais. Serait-ce une interprétation iconographique de l'idée que les patrouilles navales portugaises barrent l'accès au Brésil pour les Français?

En tout cas de tous les navires c'est un vaisseau français qui occupe une position privilégiée : il est le plus grand de tous, le mieux détaillé, placé presque au milieu de l'espace aquatique entourant le Brésil ; ses voiles sont gonflés et créent le sentiment d'un mouvement rapide. Ce vaisseau se dirige au Brésil, sa proue est tournée vers la côte.

Une telle position privilégiée du navire français par rapport aux autres correspond parfaitement à la tonalité générale du "Discours d'un grand capitaine " qui est un pamphlet ardent en faveur du commerce français au Brésil ${ }^{27}$, très mal vu par les Portugais. On sait que la présence française au Brésil dans les années 1530-1540 a été importante, y compris à cause du faible développement de la colonie par la couronne portugaise; c'est cette présence française qui incitait dans beaucoup de cas les Portugais à fortifier la côte et y créer le réseau des points forts. Cependant plusieurs vides et lacunes dans ce maillot permettaient aux Français de continuer à visiter le Brésil - pour trafiquer avec les indigènes ou pour courir sus aux navires portugais si une occasion se présentait. La crainte permanente des Portugais était qu'un beau jour les Français viendraient au Brésil pour le "peupler", mais à part d'un bruit qui courrait sur le voyage de Giovanni Verrazano, florentin au service de François I ${ }^{\mathrm{er}}$, au début des années $1520^{28}$ et une tentative décrite par baron de Saint-Blancard par rapport au voyage de son navire "Pèlerine » à la fin de cette même décennie ${ }^{29}$, on ne connaît pas d'exemples d'une création des noyaux forts permanents par les Français au Brésil avant la fondation de la France Antarctique dans la baie de Guanabara en 1555.

31 L'écho de toutes ces tendances se voit bien dans le « Discours d'un grand capitaine » et sur la carte de Gastaldi. On a déjà évoqué le fait que le cartographe ne montre pas de traces des installations européennes sur le littoral, à part l'inscription « Fernambuc » sans complément picturale et un certain pictogramme au dessus du Cap Saint-François, dépourvu d'une légende. De la même façon Pierre Crignon affirme qu' "on ne rencontre, le long de [la] côte aucune forteresse ou château qui indique la présence des Portugais, Français ou Espagnols », ayant cité ci-dessus l'exemple de Fernambuc comme exception.

Le véritable « champ de rivalité » franco-portugaise au Brésil - aussi bien sur la carte que dans le texte - se place ainsi dans une autre dimension, celle du droit de visiter le Brésil. Les Français le réclament, les Portugais ne le gardent que pour soi. Le texte du "Discours" témoigne des arguments les plus répandus des Français dans cette discussion.

Pierre Crignon développe ses propos en défense du droit de visiter le Brésil sur deux plans. Le premier se réfère au droit du découvreur et à la légitimité liée à la priorité de la découverte. D'un côté, l'auteur français attaque les Portugais sur ce champ; d'autre 
côté il est contraint de lutter avec les mêmes armes. Ainsi, il proteste contre la situation où

aussitôt que les Portugais ont navigué le long d'une côte, ils s'en emparent et la considèrent comme leurs conquête, conquête facile et à peu de frais, car elle n'a nécessité ni assaut, ni résistance ${ }^{30}$.

D'autre côté, ne pouvant pas nier complètement le droit du découvreur avancé par le Portugal, Pierre Crignon l'étend et en fait bénéficier également ses compatriotes :

partie de cette terre du Brésil fut d'abord découverte par les Portugais, et il y a environ trente-cinq ans. Denis de Honfleur découvrit l'autre partie il y a vingt ans et depuis beaucoup d'autres navires français y ont abordé, sans y rencontrer aucune trace de la domination portugaise. Aussi les habitants sont parfaitement libres et ne reconnaissent ni puissance royale ni lois ; ils aiment plus les Français ${ }^{31}$.

La déclaration de Crignon au sujet de la découverte d'une partie du littoral brésilien par les Français n'est pas un exemple unique. A la fin des années 1520 le même argument a été avancé par les Bretons dont les navires ont été interceptés par les Portugais au $B$ Bésil ${ }^{32}$; quelques décennies plus tard l'auteur anonyme du «Discours des Normands pour le trafique des Indes » constatera avec amertume qu' « ont été lesdits Français les premiers qui ont en partie découvert ce que aujourd'hui les autres possèdent $»^{33}$. En 1582 l'historien protestant Lancelot Voisin de La Popelinière évoque cette idée en regrettant que les Normands et les Bretons n'ont pas eu «l'esprit ni discrétion de laisser un seul écrit public pour assurance de leurs desseins aussi hautains et généreux que les autres ", faute de quoi c'est le Portugal, supérieure à toutes les nations en théorie et pratique des découvertes maritimes, s'est attribué «l'avantage d'être...

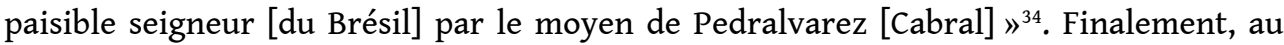
$\mathrm{XVII}^{\mathrm{e}}$ siècle l'idée de la priorité française dans la découverte de certaines parties du littoral brésilien est attestée chez le jésuite G. Fournier, l'auteur d'une «Hydrographie ${ }^{35}$ et chez le voyageur François Pyrard de Laval ${ }^{36}$.

En revenant à l'argumentation de Crignon et aux modalités de sa représentation sur la carte de Gastaldi, notons que l'enjeu du «Discours » est d'acclamer le droit des Français de visiter le Brésil plutôt que de s'y établir d'une façon permanente. Ainsi la carte de Gastaldi évoque la présence des Européens avec leurs navires et drapeaux en dehors des contours du pays. Crignon insiste sur l'ancienneté du trafic franco-indien et met en avant la thèse de l'absence de domination portugaise dans les régions fréquentées par les Français. La familiarité des Français avec certaines zones du littoral se forge donc en l'absence de tout obstacle.

39 Ces arguments doivent être placés dans le contexte de la controverse franco-portugaise au sujet de la liberté des mers : les Portugais reconnaissaient en principe la liberté de la navigation pour tous sur les mers connues, mais les autres, disaient-ils, n'étaient pas libres. Au contraire, la thèse française revendiquait la liberté de navigation sur toutes les mers sans exception ni restriction; le droit co-extensif à cette liberté était celui de trafiquer avec les Indiens du Brésil ${ }^{37}$.

Cette considération nous emmène à examiner la représentation de ce commerce franco-indien aussi bien sur la carte de Gastaldi que dans le «Discours d'un grand capitaine ». 


\section{Le commerce de troc}

41 A l'exemple d'autres cartographes de l'époque, Gastaldi insère dans la carte du Brésil des scènes de genre ; elles remplissent des zones intérieures du pays qui restent encore inconnues. On y voit les Amérindiens se reposer sous les arbres, dans les hamacs ou à l'intérieur des maisons, tirer à l'arc, couper les arbres et transporter les troncs. Les arbres en question sont le célèbre bois rouge, pau brasil, qui attirait tant les Européens et qui était à leurs yeux une des principales richesses du Brésil. Pau brasil servait pour extraire une matière colorante et peindre ensuite les tissus ou préparer des couleurs, les encres de couleur, des laques etc.; d'autre côté, les ébénistes utilisait le bois rouge en arts décoratifs. Dans leur état naturel les arbres n'avaient rien de spécial qui indiquerait leurs qualités tinctoriales; la couleur rouge du bois se révélait lorsqu'on enlevait l'écorce du tronc. Cependant sur certaines images et cartes du XVI ${ }^{e}$ siècle on dessinait les arbres aux troncs rouges pour évoquer le bois du Brésil. Dans la version colorée de la carte de Gastaldi (l'exemplaire de l'USP) ce procédé n'est pas utilisé ; pourtant une distinction « coloristique » dans la représentation des arbres ordinaires et les troncs des arbres de brésil peut être observée sur les différentes cartes de Brésil de la première moitié du XVI ${ }^{e}$ siècle ${ }^{38}$. On utilise ce moyen non seulement dans les cartes mais aussi dans d'autres documents iconographiques: ainsi, une image française des années 1550 montre bien les troncs rouges des arbres qui devaient symboliser le bois du Brésil lors d'un spectacle donné à Rouen en 1550 à l'occasion de l'entrée solennelle d'Henri II ${ }^{39}$.

Le bois rouge était un de principaux articles d'échange dans le commerce de troc des Amérindiens avec les Européens. Ceux-ci s'étonnaient du fait que les indigènes ignorent les métaux et l'usage de la monnaie; ainsi les objets pour lesquels les Européens recevaient le bois rouge étaient le plus souvent des ferrements. Les Indiens, en revanche, devaient couper les arbres, ôter des branches, transporter les troncs vers le lieu où étaient ancrés les navires et même participer au chargement.

Le "Discours d'un grand capitaine » décrit les habitants du Brésil qui échangent des bois précieux contre de petites haches, des coins de fer et des couteaux. Dans quelques contrées les habitants sont obligés d'aller chercher les bois d'échange souvent à trente lieus à l'intérieur du pays...Tous portent leur pièce de bois jusqu'à la côte, où ils la livrent aux Français, moyennent quelques petites haches, quelques coins, couteaux et objets en fer ${ }^{40}$.

De la même façon on voit sur la carte de Gastaldi plusieurs personnages qui coupent le bois (deux indigènes dans la partie gauche, un personnage au centre et son voisin muni d'une serpe avec laquelle il coupe les branches). Pas moins de quatre autres personnages portent les troncs sur leurs épaules; les troncs seuls ou rassemblés dans des lots gisent sur le sol. Pour animer la scène, le peintre y introduit des oiseaux (de toute évidence des perroquets) qui se posent sur les troncs laissés au sol ou sont perchés sur le tronc transporté par un Indien ${ }^{41}$.

45 Les efforts considérables employés par les Indiens que l'on récompensait ensuite par un outil en fer de petite valeur donnaient aux auteurs français du XVI ${ }^{\mathrm{e}}$ siècle un prétexte pour réfléchir à cette incohérence. Les opinions étaient les plus variées; ainsi, Pierre Crignon statue que «ces peuples font plus grand cas d'un clou que d'un écu » ${ }^{42}$, le voyageur André Thevet pense que les Indiens se donnent tant de peine pour l'amour qu'ils ressentent envers les chrétiens : ils les « aiment, chérissent et honnorent » ${ }^{43}$; le 
capucin Yves d'Evreux au début du XVII ${ }^{\mathrm{e}}$ siècle loue, au contraire, la sagesse des Indiens qui reconnaissent une valeur concrète et réelle d'un ferrement au lieu de s'éblouir des fausses valeurs et du luxe incarnés dans les pierres précieuses ${ }^{44}$.

En comparant le texte du «Discours » cité ci-dessus et la carte de Gastaldi, on voit que le cartographe suit soigneusement les indications de Pierre Crignon. Ainsi, dans la partie centrale les personnages coupant le bois sont placés à l'intérieur, tandis que ceux qui portent les troncs envers les marchands français sont représentés près de la côte. Ils forment tous un groupe à part ; cinq personnages, hommes, femmes et animaux se dirigent de gauche à droite (soit regardent à droite); au bout de ce trajectoire ils sont rencontrés par deux marchands français. La position de ceux-ci n'est pas choisie au hasard ; l'Indien qui tend la main aux Français et un marchand répondant à ce geste sont placé sur la côte entre les inscriptions «R. Real », «R. de S. Francisco ", «C. de S. Augustin ». Or, c'est précisément la région le plus visitée par les Français, d'après le " Discours d'un grand capitaine »:

La partie la plus fréquentée par les Français et les Bretons est située entre le cap SaintAugustin et le Port-Real, qui est placé au $12^{\mathrm{e}}$ degré ; c'est aussi dans cette partie que ce trouvent les meilleurs bois du Brésil et en plus grande quantité ${ }^{45}$.

Il est intéressant de rencontrer les mêmes indications topographiques dans les ouvrages de la seconde moitié du XVI ${ }^{\mathrm{e}}$ siècle et même au XVII ${ }^{\mathrm{e}}$. Citons quelques exemples en ordre chronologique. Sur une carte de l'Amérique de l'atlas "Theatrum orbis terrarum » d'Abraham Ortelius (première édition 1570) on distingue l'inscription "Porto Real ad quem Galli mercatum navigant » ${ }^{46}$ («Port Real où vont les marchands français ») précisément à la hauteur des fleuves Rio de São Francisco, Rio Real et Rio de São Agostinho. L'historien protestant La Popelinière, évoqué ci-dessus, affirme en 1582 que « les Français toutefois, Normands surtout \& les Bretons maintiennent... d'ancienneté trafiquer avec les sauvages du Bresil contre la rivière Saint François au lieu qu'on a depuis appelé Port Réal » ${ }^{47}$. Le jésuite Père Fournier écrit en 1643 que "Normands et Bretons maintiennent... de long temps [avoir] trafiqué sur la rivière de St. François d'où ils apportent le bois qu'ils appellent Brésil, propre pour les teintures... ${ }^{48}$.

La carte de Gastaldi et le "Discours d'un grand capitaine " qu'elle accompagne se présentent ainsi comme des initiateurs d'une tradition qui se poursuivra dans la seconde moitié du XVI ${ }^{e}$ siècle et au XVII .

\section{Conclusion}

L'analyse des informations contenues dans la carte de Gastaldi à l'appui du « Discours d'un grand capitaine » qu'elle illustre montre que les divergences entre ces documents sont moins nombreuses que les points de similitudes. La carte de Gastaldi est plus riche en toponymes et présente non seulement la côte mais aussi une zone à l'intérieure du continent où naissent les deux grands fleuves sud-américains, tandis que le texte parle du Brésil en termes extensifs en y incluant tout le cône sud de l'Amérique et ne concerne que le littoral. En revanche, le cartographe suit de près l'écrivain dans la question du développement urbain au Brésil : «Fernambuc» considéré par Crignon comme l'unique point fort portugais en 1539 (où même en 1529, à la date de voyage de Parmentier) est représenté en cette qualité par Gastaldi en 1556, sans parler des éditions suivantes, ce qui était déjà un anachronisme. 
51 ses informations par les moyens iconographiques. Ainsi, l'importance que l'auteur du «Discours d'un grand capitaine » prête à la liberté du commerce des Français avec les indigènes se traduit sur la carte par l'abondance des scènes de genre se référant au découpage et au commerce du bois rouge et par la position privilégiée du navire français par rapport aux autres vaisseaux. Même la place sur la carte où sont représentés les marchands français est choisie en accord avec les références spatiales contenues dans le texte.

Tenant compte du fait que le troisième volume des «Navigations et voyages" de Ramusio a connu trois éditions, on peut supposer que cette popularité a contribué à la propagation $\mathrm{du}$ "regard français sur le Brésil». Sans statuer assurément que les informations sur le Brésil dans les ouvrages géographiques de synthèse, tels les livres de La Popelinière ou du Père Fournier, proviennent précisément de Ramusio (bien que certains indices indirects semblent le montrer ${ }^{49}$ ), on ne peut pas nier la similitude de certaines thèses qu'on y rencontre avec les données de Crignon illustrées par Gastaldi.

\section{BIBLIOGRAPHIE}

ANTHIAUME A. Cartes marines. Constructions navales. Voyages et découvertes chez les Normands (1500 - 1650). Paris, Dumont, 1916, vol. 1.

BARNES, J. Randall. Giovanni Battista Ramusio and the history of discoveries: An analysis of Ramusio's commentary, cartography, and imagery in "Delle Navigationi et Viaggi". Arlington : The University of Texas at Arlington, 2007

BIAGGI, LECA De \& DROULERS M. Cartographie et formation territoriale. In : Cahiers des Amériques latines № 34, 1991, p. 42 - 4

BROC N. La géographie de la Renaissance. Paris, Editions du CTHS, 1986

CORTESÃO J. Historia do Brasil nos velhos mapas. Rio de Janeiro : Instituto de Rio Branco, 1965, vol. 1

COSGROVE D. Mapping New Worlds: Culture and Cartography in Sixteenth-Century Venice. In: Imago Mundi, Vol. 44 (1992)

HORODOWICH L. Armchair Travelers and the Venetian Discovery of the New World. In: The Sixteenth Century Journal, Vol. 36, No. 4 (Winter, 2005)

JULIEN Ch.-A., Les débuts de l'expansion et de la colonisation française (XV ${ }^{\mathrm{e}}-\mathrm{XVI}^{\mathrm{e}}$ siècles). Paris : PUF, 1948

MARTINIERE G. Le Brésil, terre d'enjeux de la colonisation européenne des temps modernes (XVI - XVIII ${ }^{e}$ siècle). In : MERIAN J. Y. (dir.) Les aventures des Bretons au Brésil à l'époque coloniale. Rennes :Les Portes du Large, 2007

MOLLAT M. Le commerce maritime normand à la fin du Moyen Age. Paris : Plon, 1952

Terra Brasilis, 2 | 2013 
MOLLAT M., HAUBERT J. Voyages et découvertes. Giovanni et Girolamo Verrazano navigateurs de François ${ }^{\mathrm{er}}$. Dossiers de voyages établis et commentés par M. Mollat du Jourdin et J. Haubert. Paris : Imprimerie nationale, 1982

MONTAIGNE J.-M. Le trafic du Brésil. Navigateurs Normands, Bois Rouge et Cannibales pendant la Renaissance. Rouen : ASI Communications, 2000

PARKS G. B. Ramusio's Literary History. In: Studies in Philology [University of North Carolina Press], Vol. 52, No. 2 (Apr., 1955)

PARKS G.B. The contents and sources of Ramusio's Navigationi. In: RAMUSIO Giovanni Battista. Navigationi et viaggi. Amsterdam, Theatrum Orbis Terrarum 1967-1970, vol. 1, p. 1-37.

VERGE-FRANCESCHI M. Chronique maritime de la France d'Ancien Régime. Paris, 1998 WOODWARD D. (ed). Cartography in the European Renaissance. Chicago-London, The University of Chicago Press, 2007, vol. 1-2.

\section{ANNEXES}

\section{Documentation}

Carta d'El Rey João III. In : GAMA, J.F. Fernandes. Memorias historicas da provincia de Pernambuco. Pernambuco: Typographia de M. F. de Faria, 1844

Discours des Normands pour le trafic aux Indes. In : ANTHIAUME A. Cartes marines. Constructions navales. Voyages et découvertes chez les Normands (1500 - 1650., Paris, Dumont, 1916, vol. 2, p. 565.

ESTANCELIN L. Recherches sur les voyages et découvertes des navigateurs normands en Afrique, dans les Indes orientales et occidentales. Paris : édition de Gérard Monfort, 1832

EVREUX Y. d'. Suite de l'Histoire des choses plus mémorables advenues en l'île de Maragnan es années 1613 et 1614. In : Evreux Y. d', Clastres H. (éd.). Voyage au nord du Brésil fait en 1613 et 1614, Paris, Payot, 1985

FOURNIER G. Hydrographie contenant la théorie et la pratique de toutes les parties de la navigation, composée par père Georges Fournier de la Compagnie de Jésus ; seconde édition, à Paris chez Jean Dupuis, MDCLXVII

GUENIN E. Ango et ses pilotes. D'après les documents inédits tirés des archives de France, de Portugal et d'Espagne. Paris : Imprimerie nationale, 1901

HOFFMAN B. G. Account of a Voyage Conducted in 1529 to the New World, Africa, Madagascar, and Sumatra, Translated from the Italian, with Notes and Comments. In: Ethnohistory, vol. 10, № 1 (Winter, 1963)

LE TESTU G. Côtes du Brésil ; Océan Atlantique Sud entre l'Afrique occidentale et le Brésil In : Cosmographie universelle selon les navigateurs, tant anciens que modernes, 1556, $\mathrm{f}$. XLV, XLVI. - Bibliothèque de Vincennes.

LA POPELINIERE. Les trois mondes. Edition établie et annotée par A.-M. Beaulieu. Genève : Librairie Droz, 1997

LERY J. de. Histoire d'un voyage fait en la terre du Brésil (1578, seconde édition - 1580) / Texte établie, présenté et annoté par F. Lestringant. Paris., 1999 
NOTHNAGLE J. Pierre Crignon : poète et navigateur. CEuvres en prose et en vers, présentées et annotées par J. Nothnagle. Birmingham (Alabama) : Summa publications, 1990

ORTELIUS A. Theatrum orbis terrarum (1570). Atlas, Museu das Belas Artes Pouchkine (GMII), Moscou, département de graphique, inv. № 1601

[PYRARD DE LAVAL, F.] Voyage de Pyrard de Laval aux Indes orientales (1601 - 1611), contenant sa navigation aux Maldives, Moluques, Brésil; les divers accidents, aventures et dangers qui lui sont arrivés en ce voyage, tant en allant et retournant, que pendant son séjour de dix ans en ces pays-là. Paris : Editions Chandeigne, 1998, vol. 2

RAMUSIO G. B. Terzo Volume delle Navigationi et Viaggi, nel quale si contengono le Navigationi al Mondo Nuovo, à gli Antichi incognito, fatte da Christoforo Colombo Genovese, [...] \& accresciuti poi da Fernando Corteze, da Francesco Pizarro, \& altri valorosi Capitani, in diverse parti delle dette Indie, in nome di Carlo V imp [...], Venetia, 1556

Relações das [cartas] patentes que mandou João da Silveira do a que veio o Rey d'armes [Helies Alesgle d'Angoulême], [1528?]. In: Bibliothèque nationale de France, Nouvelles Acq. Françaises, 9386, f. 104

SANTAREM, visconde de. Quadro elementar das relações políticas e diplomáticas de Portugal com as diversas potencias do mundo, desde o principio da monarquia portuguesa até os nossos dias. Paris, 1843, tomo 3

THEVET A. La Cosmographie universelle d'André Thevet cosmographe du Roi, illustrée de diverses figures des choses les plus remarquables vues par l'auteur et inconnues de nos Anciens et Modernes. In : Les Français en Amérique pendant la seconde moitié du XVI ${ }^{e}$ siècle. Paris : Presses universitaires françaises, 1953

\section{NOTES}

1. option=com_jumi\&fileid=14\&Itemid=99\&idMapa=579]

http://www.cartografiahistorica.usp.br/index.php?

2. RAMUSIO G. B. Terzo Volume delle Navigationi et Viaggi, nel quale si contengono le Navigationi al Mondo Nuovo, à gli Antichi incognito, fatte da Christoforo Colombo Genovese, [...] \& accresciuti poi da Fernando Corteze, da Francesco Pizarro, \& altri valorosi Capitani, in diverse parti delle dette Indie, in nome di Carlo V imp [...]. Venetia, Nella Stamperia de Giunti, 1556; 1565; 1606.

3. SKELTON R.A. Introduction. In: RAMUSIO Gian Batista. Navigationi et viaggi. Amsterdam, Theatrum Orbis Terrarum 1967-1970, vol. 1, p. VI, VII.

4. Ibidem.

5. Ibid, p. XII.

6. COSGROVE D. Mapping New Worlds: Culture and Cartography in Sixteenth-Century Venice. In: Imago Mundi, Vol. 44 (1992), p. 74.

7. Le texte auquel nous allons nous référer ici provient de l'édition de 1556 (conservé à la bibliothèque de Heidelberg): Terzo Volume delle Navigationi et Viaggi, nel quale si contengono le Navigationi al Mondo Nuovo, à gli Antichi incognito, fatte da Christoforo Colombo Genovese, [...] \& accresciuti poi da Fernando Corteze, da Francesco Pizarro, \& altri valorosi Capitani, in diverse parti delle dette Indie, in nome di Carlo V imp [...], Venetia In Venetia nella stamrepia de Giunti, l'anno MDLVI. Signalons deux importantes éditions modernes fax-similées :

RAMUSIO Gian Batista. Navigationi et viaggi. Venice, 1563-1606. With an introd. by R. A. Skelton and an analysis of the contents by George B. Parks. Amsterdam: Theatrum Orbis Terrarum, 
1967-1970, 3 vols ; RAMUSIO Gian Batista. Navigationi et viaggi. A cura di Marica Milanesi. Torino: G. Einaudi, 1978, 6 vols.

Il existe également des traductions de l'italien :

En français :

- Discours d'un grand navigateur du port de Dieppe en France, sur les voyages faits aux terres nouvelles des Indes Occidentales, dans la partie appelée la Nouvelle France, depuis le $40^{\circ}$ jusqu'au $47^{\mathrm{e}}$ degré, sous le pôle arctique, aux terres du Brésil, de la Guinée et aux îles de Saint Laurent et de Sumatra, jusqu'où sont parvenus les caravelles françaises. In : ESTANCELIN L. Recherches sur les voyages et découvertes des navigateurs normands en Afrique, dans les Indes orientales et occidentales. Paris : édition de Gérard Monfort, 1832, pp. 216-240.

- Discours d'un grand capitaine de mer français du lieu dit de Dieppe sur les navigations faites à la terre neuve des Indes Occidentales, nommé la Nouvelle France, [s'étendant] de $40^{\circ}$ jusqu'à $47^{\circ}$ sous le pôle arctique, et sur la terre du Brésil, de Guinée, de l'île Saint-Laurent et de celle de Sumatra, jusqu'où ont navigué les caravelles et les navires français. In : Pierre Crignon : poète et navigateur. Cuvres en prose et en vers, présentées et annotées par J. Nothangle. Birmingham (Alabama) : Summa publications, 1990, pp. $95-113$.

En anglais:

HOFFMAN B. G. Account of a Voyage Conducted in 1529 to the New World, Africa, Madagascar, and Sumatra, Translated from the Italian, with Notes and Comments. In: Ethnohistory, vol. 10, № 1 (Winter, 1963), p. 1 - 79 (Disponible sur [http://www.jstor.org/stable/480388]). Cette publication contient également une reproduction en fac-similé d'une partie du « Discours ».

8. Discorso d'un gran capitano...In: Terzo volume delle Navigationi et Viaggi, p. 417v.

9. Sur l'expérience française de Ramusio voir PARKS G. B. Ramusio's Literary History. In: Studies in Philology [University of North Carolina Press], Vol. 52, No. 2 (Apr., 1955), p. 129-130.

10. SKELTON R.A. Op.cit., p. IX.

11. "Questo discorso ci é parso veramĕte molto bello, \& degno di esser letto da ogni uno, ma ben ci dolemo di non sapere il nome dell'auttore, pcioche nõ nonendo il suo nome ci par di fare ingiuria alla memoria di cosi valěte, \& gĕtil caualiero.». - Discorso d'un gran capitano...In: Terzo volume delle Navigationi et Viaggi, p. $417 \mathrm{v}$.

12. ESTANCELIN L. Op. cit., p. 189.

13. «[un Grã Capitano Frãcese] descriue il viaggio, che si fa alla terra nuoua dell'Indie Occidentali, che hora chiamano la nuoua Frãcia, \& ancho alla terra del Brasil pur delle dette Indie, Guinea, costa delle Meleghette sopra l'Africa, doue tutto il giorno li Frãcesi pratticano cõ lor navi. il sopradetto Capitano poi con due naui armate in Dieppa di Normandia, volse andar fino all'Isola Taprobana in Leuante hora detta Sumatra, doue cõtrattto con quei popoli, \& carico di specie ritornó à casa.» - Discorso d'un gran capitano... In: Terzo volume delle Navigationi et Viaggi, p. $417 \mathrm{v}$; la traduction en français est donnée d'après NOTHAGLE J. Op. cit., p. 111.

14. Discorso d'un gran capitano...In: Terzo volume delle Navigationi et Viaggi, p. 417v.

15. Une partie du recueil en question est intitulée « Déploration sur la mort desditz Parmentiers composée par Pierre crignon, compagnon desditz Parmentiers en ladicte nauigation ». La mention de la dernière page du livre donne comme date d'impression le 7 janvier 1531 (à l'époque le nouvel an commençait à Pâques, et il s'agit ainsi du 1532 selon le nouveau style). NOTHAGLE J. Op. cit., p. 10.

16. ESTANCELIN L. Op. cit., p. 230. Ainsi, selon le « Discours... », la date de la découverte du Brésil est 1504 ; cette idée n'était pas rare au $\mathrm{XVI}^{\mathrm{e}}$ siècle où certaines cartes géographiques au rayonnement international affichaient une légende "Bresilia a Lusitanis $A^{\circ} 1504$ inventa ». ORTELIUS A. Theatrum orbis terrarum (1570). Atlas, Musée des Beaux Arts Pouchkine (GMII), Moscou, département de graphique, inv. № 1601. Une variante numériisée est disponible sur le 
site de la Library of Congress: [http://www.loc.gov/resource/g3200m.gct00003/\#seq-2]. Cette datation renvoie au second voyage d'Amerigo Vespucci.

17. Cf. ANTHIAUME A. Cartes marines. Constructions navales. Voyages et découvertes chez les Normands (1500 - 1650). Paris, Dumont, 1916, vol. 1, p. 460 ; JULIEN Ch.-A. Les Français en Amérique pendant la première partie du XVI ${ }^{e}$ siècle. Paris : PUF, 1946, p. 24.

18. PARKSJ.B. The contents and sources of Ramusio's Navigationi. In: RAMUSIO Gian Batista. Navigationi et viaggi. Amsterdam, Theatrum Orbis Terrarum 1967-1970, vol. 1, p. 35.

19. Ibid, p. 1.

20. Pour l'exemplaire de 1556 nous avons consulté celui de la bibliothèque de Heidelberg [http:// digi.ub.uni-heidelberg.de/diglit/ramusio1556bd3/0936]; pour l'exemplaire de 1565 - celui de la la Bibliothèque nationale $\mathrm{du}$ Brésil [http://objdigital.bn.br/acervo_digital/div_cartografia/ cart395872.jpg] et pour l'exemplaire de 1606 - celui de la Bibliothèque nationale de France [http://gallica.bnf.fr/ark:/12148/bpt6k131851r/f791.image].

21. Discorso d'un gran capitano...In: Terzo volume delle Navigationi et Viaggi, p. 426r.

22. Cf. les cartes du Brésil par Guillaume Le Testu (1556) :

LE TESTU G. Côtes du Brésil ; Océan Atlantique Sud entre l'Afrique occidentale et le Brésil In : Cosmographie universelle selon les navigateurs, tant anciens que modernes, 1556, $\mathrm{f}$. XLV, XLVI. - Bibliothèque de Vincennes (exemplaire digitalisé est disponible sur http://catalogue.bnf.fr/ark:/12148/cb42467457f). Reproduction en couleur: MONTAIGNE J.-M. Le trafic du Brésil. Navigateurs Normands, Bois Rouge et Cannibales pendant la Renaissance Rouen : ASI Communications, 2000, p. 17.

23. Sur la thèse de l'insularité du Brésil voir : CORTESÃO J. Historia do Brasil nos velhos mapas. Rio de Janeiro : Instituto de Rio Branco, 1965, vol. 1; LECA De BIAGGI E., DROULERS M. Cartographie et formation territoriale. In : Cahiers des Amériques latines № 34, 1991, p. 42 - 43 ; MARTINIERE G. Le Brésil, terre d'enjeux de la colonisation européenne des temps modernes (XVI $-\mathrm{XVIII}^{\mathrm{e}}$ siècle). In : MERIAN J. Y. (dir.) Les aventures des Bretons au Brésil à l'époque coloniale. Rennes :Les Portes du Large, 2007, p. 27.

24. «A lungo questa costa cosi verso ponente, come mezzo di, non vé alcuna fortezza ne castello per li Portoghesi, slvo vn luogo detto Fernanbuch, il qual è appresso capo di santo Agostino, dove è certe piccole fortezze di legname con alcune poche gĕte bãdite di Portogallo».- Discorso d'un gran capitano...In: Terzo volume delle Navigationi et Viaggi, p. 426r ; la traduction française est donnée d'après ESTANCELIN L. Op. cit., p. 228.

25. Ainsi, vers le milieu des années 1550 sur la côte du Brésil on comptait déjà São Vicente (1532), Porto Seguro (1535), Ilheus (1536), Santos (1536), Salvador (1549), Espírito Santo (1551).

26. BROC N. La géographie de la Renaissance. Paris, Editions du CTHS, 1986, p. 43.

27. Discorso d'un gran capitano...In: Terzo volume delle Navigationi et Viaggi, p. 426v.

28. «1522. Nesta data sendo El Rei avisado por alguns Portuguezes que negociavão em França que certo Florentino, por nome João Verezano, se havia offerecido a Francisco I Rei de França, para descobrir no Oriente novos reinos, e para irem povoar o Brasil, se fazião nos portos de Normandia prestes varias armadas com o favor dos Almirantes das costas de França, e dissimulação do dito Francisco I, juntando-se a isto as quiexas que em Portugal havia pelos damnos que se experimentavão da parte dos corsarios francezes..." - SANTAREM, visconde de. Quadro elementar das relações políticas e diplomáticas de Portugal com as diversas potencias do mundo, desde o principio da monarquia portuguesa até os nossos dias. Paris, 1843, tomo 3, p. 197-198.

29. Sur le voyage de la "Pèlerine" et la tentative de dresser un fort français au Brésil voir: Protestation de Bertrand d'Ornessan, baron de Saint-Blancard, commandant les galères du roi dans la Méditerranée, contre la prise du navire «La Pèlerine». In : GUENIN E. Ango et ses pilotes. D'après les documents inédits tirés des archives de France, de Portugal et d'Espagne. Paris: Imprimerie nationale, 1901, p. 256-261 (l'original en latin) et p. 42-47 (traduction de la partie principale du 
document en français).

Cette tentative n'est pas restée sans connaissance des Portugais, comme en témoigne une lettre du roi Jean III de Portugal à Martim Afonso du 28 septembre 1532: "Na costa de Andaluzia foi tomada agora pelas minhas caravelas que andavam na armada do Estreito, uma nau francesa carregada de brasil, e trazida nesta cidade a cual foi de Marselha a Pernambuco, e desembarcou gente em terra, e desfez uma feitoria mihna que ahi estava, e deixou la 70 homens com tenção de povoarem a terra e de se defenderem...”. Carta d'El Rey João III. In: GAMA, J.F. Fernandes. Memorias historicas da provincia de Pernambuco. Pernambuco: Typographia de M. F. de Faria, 1844, vol.1, p. 59-61.

30. «Poscia che esti hanno nauigato al lungo d'una costa, esti se la fanno tutta sua. Ma tal conquista è molto facile à fare \& senza grã spesa, perche non vi sono assalti, ne resistentia». Discorso d'un gran capitano... In: Terzo volume delle Navigationi et Viaggi, p.426v ; la traduction française est donnée d'après ESTANCELIN L. Op. cit., p. 231-232.

31. "Questa terra del Brasil fu primamente scoperta da Portoghesi in qualche parte, \& sono circa trentacinque anni. L'altra parte fu scoperta per uno de Honfleur chiamato Dionisio di Honfleur da venti anni in qua. \& di poi molti altri nauilij di Francia vi sono stati, \& mai non trouorono Portoghesi in terra alcuna che la tenessero per il Re di Portogallo. \& quelli della terra sono liberi, \& non soggetti ne à Re, ne à legge, \& amano piu li Francesi». - Discorso d'un gran capitano... In: Terzo volume delle Navigationi et Viaggi, p. 426v; la traduction française est donnée d'après ESTANCELIN L. Op. cit., p. 230.

32. "A El-Rey nosso soberano senhor e a meus senhores do seu conselho Supricam humilmente Yvon de Cretugar, Frances [François] Guerret, Mathurin Fournemouche, Joham Bureau e Joham Jamet, mercadores de vossa terra e ducado de Bretanha, vossos muito humildes servidores e sujeitos, como os ditos supricantes que são mercadores frequentando os mares e muitas diversas terras e entre outras as terras do Brasil que são muito grandes, as quaes os bretões descubriram e per alguns lugares e os portugueses per outros lugares...". - Relações das [cartas] patentes que mandou João da Silveira do a que veio o Rey d'armes [Helies Alesgle d'Angoulême], [1528?]. In: Bibliothèque nationale de France, Nouvelles Acq. Françaises, 9386, f. 104.

Il est à noter que l'épisode avec la prise de trois navires bretons au Brésil en 1527 qui a donné lieu à un litige (lors duquel une prétention à la priorité de la découverte d'une partie du Brésil a été formulée) a été décrit dans les documents dont les originels français ne sont pas parvenus à nos jours et que nous connaissons à travers les traductions portugaises de l'époque, conservées aux archives de Torre do Tombo à Lisbonne.

La déclaration des Bretons de Saint-Pol-de Léon a connu des troubles avant que son statut d'un document réel soit établit par M. Mollat.. A la fin de XIX ${ }^{\mathrm{e}}$ le spécialiste en histoire de la marine française, Ch. de La Roncière, a indiqué la présence de sa copie portugaise dans les archives portugaises en signalant la cote du document. L'historien portugais A. Pimenta a suivi cette référence et n'a trouvé aucun témoignage du Brésil dans les documents cités qui parlaient de Moluques; cela a donné occasion à Ch.-A. Julien, éminent spécialiste en histoire coloniale de France, de s'exclamer: "Voilà comment on écrit l'histoire...Il est mieux qu'on puisse la réécrire ». - JULIEN Ch.-A. Les débuts de l'expansion et de la colonisation française (XVe-XVI ${ }^{e}$ siècles). Paris : PUF, 1948, p.17.

Seulement quelques années plus tard M. Mollat a expliqué le désaccord qui a résulté d'une erreur dans la cote d'archives et a rétablit la déclaration des Bretons dans ses droits. - MOLLAT M. Le commerce maritime normand à la fin du Moyen Age. Paris : Plon, 1952, p. 256, n.35.

L'épisode de 1527 est également examiné dans : RAMALHOSA GUERREIRO L. M. La prise de trois navires bretons sur les côtes du Brésil en 1527. In : La Bretagne, le Portugal, le Brésil : Echanges et rapports. Actes du Cinquantenaire de la création en Bretagne de l'enseignement du portugais. Paris : Les Presses du Palais Royal, 1973, pp. 103-111. 
33. Discours des Normands pour le trafic aux Indes. In: ANTHIAUME A. Cartes marines. Constructions navales.., vol. 2, p. 565.

34. LA POPELINIERE. Les trois mondes. Edition établie et annotée par A.-M. Beaulieu. Genève : Librairie Droz, 1997, p. 379, 380.

35. FOURNIER G. Hydrographie contenant la théorie et la pratique de toutes les parties de la navigation, composée par père Georges Fournier de la Compagnie de Jésus; seconde édition, à Paris chez Jean Dupuis, MDCLXVII, p.245 (la première édition date de 1643 : cf. VERGE-FRANCESCHI M. Chronique maritime de la France d'Ancien Régime. Paris, 1998, p. 36).

36. [PYRARD DE LAVAL, F.] Voyage de Pyrard de Laval aux Indes orientales (1601 - 1611), contenant sa navigation aux Maldives, Moluques, Brésil; les divers accidents, aventures et dangers qui lui sont arrivés en ce voyage, tant en allant et retournant, que pendant son séjour de dix ans en ces pays-là. Paris : Editions Chandeigne, 1998, vol. 2, p. 809.

37. MOLLAT M., HAUBERT J. Voyages et découvertes. Giovanni et Girolamo Verrazano navigateurs de François $I^{e r}$. Dossiers de voyages établis et commentés par M. Mollat du Jourdin et J. Haubert. Paris : Imprimerie nationale, 1982, p. 119.

38. Citons à titre d'exemple l'Atlas nautique du Monde, dit atlas Miller de Lobo Homem (1519), les cartes de Brésil de Jean Rotz (1542) et Nicolas Vaillard (1547). Les arbres et les arbrisseaux formant les bocages ne sont pas marqués de quelques signes distinctifs ; pourtant, les troncs que les Indiens portent sur leurs épaules au rivage sont d'un rouge intense.

Cf. l'exemplaire numérisé de l'Atlas Miller [http://catalogue.bnf.fr/ark:/12148/cb40887479k] et les reproductions en couleur dans : MONTAIGNE J.-M. Op. cit., p. 15, 38.

39. Un détail de cette miniature montrant les arbres aux troncs rouges est reproduit dans: MONTAIGNE J.-M. Op. cit., p. 32 ; BUENO E. Brasil : uma História. A Incrível saga de um pais. São Paulo, 2003, p. 23.

40. «Barattano il verzin in manarette, cunei, coltelli, \& in qualche luogo é necessario che lo vadino à cercar in compagnie fin à trente leghe dentro del paese [...] \& partano ciascun il suo pezzo di legno alli Francesi fin alla marina, \& barattano colle dette manare, cunei \& coltelli \& altri ferramenti» - Discorso d'un gran capitano...In: Terzo volume delle Navigationi et Viaggi, p. 426r ; la traduction française est donnée d'après ESTANCELIN L. Op. cit., p. 229.

41. Le même motif - des perroquets se posant sur les troncs que les Indiens apportent aux Français - peut être vu sur un relief en bois provenant d'une maison rouennaise du XVI ${ }^{\mathrm{e}}$ siècle. Rouen. Musée départemental des Antiquités. № 53. Bas-relief de bois sculpté dit de «Isle du Brésil » provenant de la façade du 17, rue Malpalu, à Rouen.

42. Discorso d'un gran capitano...In: Terzo volume delle Navigationi et Viaggi, p. 426r; la traduction française est donnée d'après ESTANCELIN L. Op. cit., p. 229.

43. THEVET A. La Cosmographie universelle d'André Thevet cosmographe du Roi, illustrée de diverses figures des choses les plus remarquables vues par l'auteur et inconnues de nos Anciens et Modernes. In : Les Français en Amérique pendant la seconde moitié du XVI siècle. Paris : Presses universitaires françaises, 1953, p. 221.

44. EVREUX Y. d'. Suite de l'Histoire des choses plus mémorables advenues en l'île de Maragnan es années 1613 et 1614. In : EVREUX Y. d', CLASTRES H. (éd.). Voyage au nord du Brésil fait en 1613 et 1614, Paris, Payot, 1985, p. $83-84$.

45. «Dal capo di santo Agostino fin al porto reale, il quale in dodici gradi. quiui è doue li Francesi \& Bretoni frequentano piu, \& doue si troua piu verzino \& migliore». - Discorso d'un gran capitano...In: Terzo volume delle Navigationi et Viaggi, p. 426r ; la traduction française est donnée d'après ESTANCELIN L. Op. cit., p. 228.

46. ORTELIUS A. Theatrum orbis terrarum. Musée des beaux arts Pouchkine (GMII), Moscou, département de graphique, inv. № 1601.

47. LA POPELINIERE. Les trois mondes,. p. 379, 380. 
48. FOURNIER G. Hydrographie contenant la théorie et la pratique de toutes les parties de la navigation..., p. 245.

49. Ainsi, dans la partie se référant à l'Amérique espagnole La Popelinière semble emprunter la liste des auteurs de l'introduction de Ramusio au troisième volume de " Navigationi et viaggi ». Serait-ce la preuve de sa connaissance de cet ouvrage, et, par conséquent, du texte de Crignon? La Popelinière n'évoque pas Ramusio comme source. -Cf. BEAULIEU A.-M. Commentaire dans LA POPELINIERE. Op. cit., p. 290.

\section{RÉSUMÉS}

L'article analyse la carte du Brésil exécutée par Giacomo Gastaldi pour un recueil important des relations de voyages Delle Navigationi et Viaggi de Giovanni Battista Ramusio (première édition 1556). Une étude de la carte se fait en comparaison avec le texte de la relation du voyage qu'elle illustre et accompagne - le Discorso d'un gran capitano di mare francese attribué a Pierre Crignon. Une démarche comparatiste consiste à révéler les similitudes et les décalages entre la carte de Gastaldi et le texte de Crignon.

$\mathrm{O}$ artigo analisa o mapa do Brasil realizado por Giacomo Gastaldi para a importante coleção de relatos de viagem titulada Delle Navigationi et Viaggi de Giovanni Battista Ramusio (por primeira vez editada em 1556). É feito um estudo do mapa em comparação ao texto da relação da viagem que ele ilustra e acompanha, o Discorso d'un gran capitano di mare francese atribuído a Pierre Crignon. Uma abordagem comparativa permitirá revelar as semelhanças e as diferenças entre o mapa de Gastaldi e o texto de Crignon.

El artículo analiza el mapa del Brasil ejecutado por Giacomo Gastaldi para la importante colección de relaciones de viaje Delle Navigationi et Viaggi de Giovanni Battista Ramusio (editada por primera vez en 1556). Es realizado un estudio del mapa en comparación al texto del relato que ilustra y acompaña, el Discorso d'un gran capitano di mare francese atribuido a Pierre Crignon. Un abordaje comparativo consiste en revelar las semejanzas y las diferencias entre el mapa de Gastaldi y el texto de Crignon.

This paper analyzes the map of Brazil made by Giacomo Gastaldi for the important collection of travel relations Delle Navigationi et Viaggi of Giovanni Battista Ramusio (first edited in 1556). The map is examined in comparison with the text of the relation it illustrates and features, the Discorso d'un gran capitano di mare francese assigned to Pierre Crignon. A comparative approach means to show the similarities and differences between Gastaldi's map and Crignon's text.

\section{INDEX}

Palavras-chave : Gastaldi, Ramusio, Crignon, Cartografia, Brasil

Palabras claves : Gastaldi, Ramusio, Crignon, Cartografía, Brasil

Index chronologique : 1556-1606

Keywords : Gastaldi, Ramusio, Crignon, Cartography, Brazil

Index géographique : Brasil

Mots-clés : Gastaldi, Ramusio, Crignon, Cartographie, Brésil 


\section{AUTEURS}

\section{OLGA OKUNEVA}

Docteur en histoire moderne (Université d'Etat de Moscou Lomonossov; Université de Paris-

Sorbonne), chargée de recherche à l'Institut d'histoire universelle de l'Académie des sciences de Russie, membre associé du Centre Roland Mousnier (Université de Paris-Sorbonne - CNRS)

olga.okouneva@gmail.com 